\title{
Tyramine Localization Closely Corelates to Circular Vesicles Within the Mouse Uterine Horn Using Correlational Fluorescence and Scanning Electron Microscopy
}

SM Bukola Obayomi ${ }^{1}$ and D Page Baluch ${ }^{2}$

${ }^{1}$ School of Life Sciences, Arizona State University, Chandler, Arizona, United States

*Corresponding author: page.baluch@asu.edu

Human preterm labor is one of the most significant issues facing women, affecting ten percent of all pregnancies [1]. Reproductive scientists are faced with the challenge of trying to understand the complex molecular and cellular signals that regulate uterine activity during human pregnancy and labor. Even though preterm labor accounts for a large portion of perinatal mortality and morbidity, there still is not an effective therapeutic strategy for the treatment or prevention of preterm labor.

The uterus is innervated with a plexus, which is a network of neurons composed of varicosity regions $(\sim 1$ micron) containing neurotransmitters. Dopamine is a neurotransmitter that has been shown to inhibit smooth muscle contractions while a recent study has found that tyramine, a biogenic amine from the same biosynthetic pathway, and its specific receptor TAAR1 (trace amine associated receptor 1), are localized within the region of the uterine plexus and can induce uterine contractions in vitro. It was also observed that there was an increased synaptic vesicle recruitment in tyramine treated uterine tissues [2]. Research in multiple mammals, including humans, has shown pinocytic vesicle formation along the lumen of the uterus which is thought to serve as uptake mechanism to reduce inner lumen volume to draw the embryo close for implantation [3]. These circular vesicles (0.5-5 microns diameter) have been named pinopods or uterodomes and have only been studied for their role at the uterine lumen but their function is not completely known. This study examines cross sections of uterine smooth muscle and found circular vesicles localized near or within the myometrium. These vesicles are similar in size, shape and appearance to both varicosities and uterodomes based on previous scanning electron micrograph findings [3]. This research identified similar circular vesicles and found localization of tyramine to these areas in and around the myometrium which varied in concentration and size based on the degree of hormonal stimulation. This research may provide a better understanding to the mechanism of how tyramine is able to modulate smooth muscle contraction and provide insight into developing a treatment to prevent premature labor.

Using fluorescent and scanning electron microscopy, mouse uterine tissue was imaged to corelate localization of tyramine to circular vesicle locations between and around muscle fibers in cross sections of the mouse uterine horn. This protocol was approved through the IACUC protocol 15-1388T at Arizona State University (ASU). The tissues were obtained from a GFP LifeAct C57BL/6 transgenic mouse line [4]. Protocols for initiating ovulation and procurement are detailed in a previous publication [5]. After fixation in formaldehyde made $4 \%$ in PBS for 48 hours, the tissues were incubated in $30 \%$ sucrose overnight. The tissues were next placed into individual molds containing Tissue-Tek O.C.T. (VWR) and quickly frozen. Tissues were thickly sectioned at 250 microns using a Leica CM1950 cryostat. The samples were labeled with primary anti-tyramine (Millipore) antibody followed by secondary antibody labeling using Alexa 568 (ThermoFisher). Fluorescent images were acquired on Leica SP8 confocal microscope housed within the School of Life Sciences at ASU (NIH SIG award 1 S10 OD023691-01). After confocal imaging, samples were processed for SEM by dehydration through a series of acetone solutions which was replaced with liquid $\mathrm{CO}_{2}$ during critical point drying. The specimen was mounted on a stub, sputter coated with gold and then imaged on the JEOL JSM 6300 SEM in the Life Sciences EM 
core facility at ASU. The confocal and SEM image files were imported into Adobe Photoshop for analysis [6].
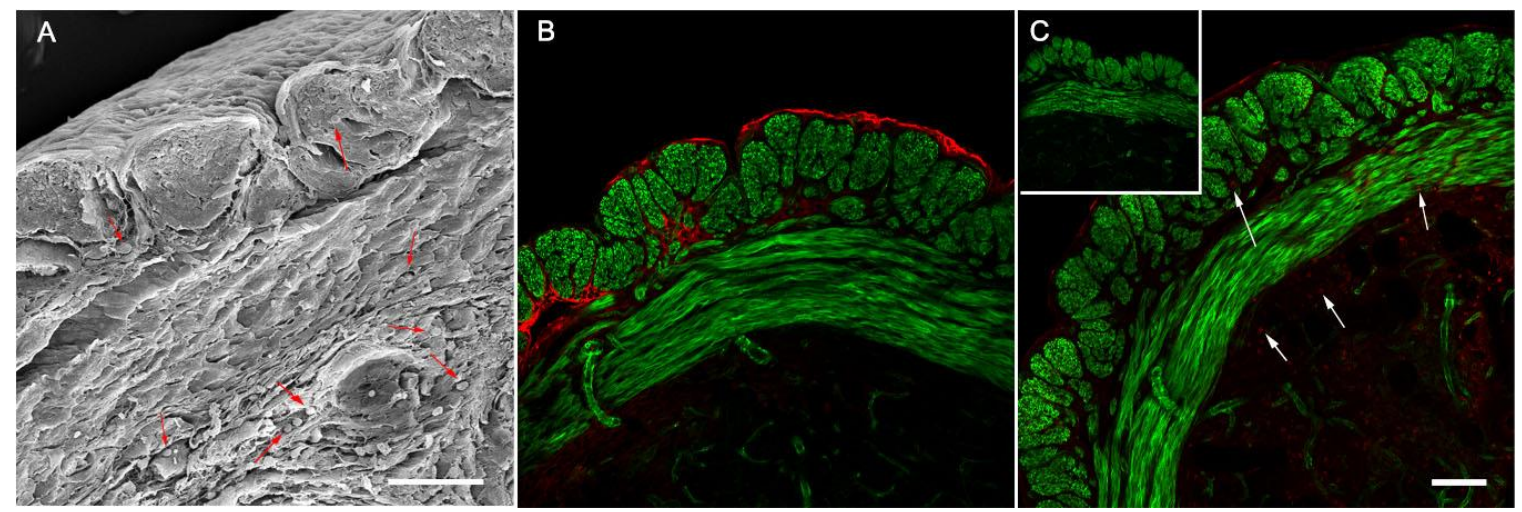

Figure 1. Localization differences of dopamine and tyramine in a cross section of the mouse uterine horn. Unstimulated uterine horn from GFP lifeact mice shows distinct localization (red) of dopamine (fig. 1b.) or tyramine (fig. 1c.) near the smooth muscle fibers (green) using confocal microscopy. The tyramine localization closely correlates to the localization of circular vesicles found within the same cross section visualized using SEM (fig. 1a.). Scale bar is 50 microns.

\section{References}

[1.] Tribe, R. M. (2001). Regulation of human myometrial contractility during pregnancy and labour: are calcium homeostatic pathways important? EXP PHYSIOL, 86(2), 247-254.

[2.] Obayomi, S. M. B., et al. (2019). Ultrastructural Modifications of the Mouse Uterus upon Biogenic Amine Stimulation. Microsc. Microanal, 25(S2), 1156-1157

[3.] Kabir-Salmani, M. et al. (2005). Secretory role for human uterodomes (pinopods): secretion of LIF. Mol Human Repro 11 (8) 553-559.

[4.] J. Riedl, et.al. Nat Methods 5 (7) (2008), 605-607.

[5.] D.P. Baluch and D.G. Capco. (2008) Dev. Biol. 317, pp. 46-58.

[6.] We acknowledge the Graduate and Professional Student Association at ASU for their generous graduate research award funding. 